\title{
Cysteine-rich secretory protein 3 overexpression is linked to a subset of PTEN-deleted ERG fusion-positive prostate cancers with early biochemical recurrence
}

Katharina Grupp ${ }^{1 *}$, Sebastian Kohl ${ }^{2 *}$, Hüseyin Sirma ${ }^{2}$, Ronald Simon ${ }^{2}$, Stefan Steurer ${ }^{2}$, Andreas Becker ${ }^{3}$, Meike Adam ${ }^{3}$, Jakob Izbicki ${ }^{1}$, Guido Sauter ${ }^{2}$, Sarah Minner ${ }^{2}$, Thorsten Schlomm ${ }^{3,4}$ and Maria Christina Tsourlakis ${ }^{2}$

${ }^{1}$ General, Visceral and Thoracic Surgery Department and Clinic, University Medical Center HamburgEppendorf, Hamburg, Germany; ${ }^{2}$ Institute of Pathology, University Medical Center Hamburg-Eppendorf, Hamburg, Germany; ${ }^{3}$ Martini-Clinic, Prostate Cancer Center, University Medical Center Hamburg-Eppendorf, Hamburg, Germany and ${ }^{4}$ Department of Urology, Section for translational Prostate Cancer Research, University Medical Center Hamburg-Eppendorf, Hamburg, Germany

The aim of this study was to determine whether cysteine-rich secretory protein 3 (CRISP3) expression is linked to clinically or molecularly relevant subgroups of prostate cancer. A tissue microarray representing samples from $>10000$ prostate cancers from radical prostatectomy specimens with clinical follow-up data were analyzed for CRISP3 expression by immunohistochemistry. CRISP3 expression was also compared with key genomic alterations of prostate cancer. CRISP3 staining was found as weak in $15 \%$, moderate in $8.5 \%$, and strong in $\mathbf{7 . 2 \%}$ of prostate cancers, whereas no expression was detected in normal prostate. Strong CRISP3 expression was linked to advanced tumor stage, high Gleason score, and positive surgical margin status $(P<0.0001$ each). There was a marked accumulation of high CRISP3 expression in PTEN-deleted ERG-positive tumors $(P<0.0001)$. A total of, $21.7 \%$ of ERG-positive and PTEN-deleted cancers had strong CRISP3 expression, but only $10.4 \%$ of ERG-positive cancers without PTEN deletion $(P<0.0001)$. The rate of high CRISP3 expression was $2.5 \%$ in $E R G$-negative cancers ( $P=0.0001$; vs $E R G$-positive cancers). Accordingly, CRISP3 overexpression was associated with early prostate-specific antigen recurrence in all tumors $(P=0.0013)$ as well as in ERGnegative $(P=0.004)$ and ERG-positive cancers $(P=0.0318)$. CRISP3 expression did not retain prognostic significance in models also involving PTEN deletions. Strong CRISP3 expression is associated with unfavorable tumor phenotype and early recurrence in prostate cancers. The tight link of strong CRISP3 expression to the ERG fusion-positive prostate cancers with PTEN deletions provides further evidence for the existence of molecularly distinct subgroups of prostate cancers.

Modern Pathology (2013) 26, 733-742; doi:10.1038/modpathol.2012.206; published online 30 November 2012

Keywords: CHD1; CRISP3; ERG; FOXP1; MAP3K7; prostate cancer; PTEN

Prostate cancer is, with about one million new cases and 250000 deaths per year worldwide, a major cause of morbidity and mortality linked to human cancer. ${ }^{1}$ As prostate cancer often has a slow disease progression and typically affects elderly men, it is

Correspondence: Dr MC Tsourlakis, Institute of Pathology, University Medical Center Hamburg-Eppendorf, Martinistr. 25, 20246 Hamburg, Germany.

E-mail: m.tsourlakis@uke.de

${ }^{*}$ These authors contributed equally to this work.

Received 23 August 2012; revised 19 October 2012; accepted 19

October 2012; published online 30 November 2012 evident, that not every patient requires potentially curative but aggressive treatment. Therapeutic options vary from active surveillance to surgical or radiation therapy. The only established pretreatment prognostic parameters currently include Gleason grade, tumor extent on biopsies, preoperative prostate-specific antigen (PSA), and clinical parameters. These data are statistically powerfull but still not sufficent for optimal treatment decisions in individual patients. There is a considerable hope, that the analysis of molecular features will better enable an individual prediction of tumor aggressiveness in the future. 
Cysteine-rich secretory proteins, including cysteine-rich secretory protein 3 (CRISP3) are poorly characterized extracellular proteins, which are preferentially expressed in cells of the reproductive tract and immune system. CRISP 3 appears to be linked to innate immunity and inflammation (reviewed in Gibbs et $a l^{2}$ ). Several studies recently suggested a role for CRISP3 in prostate cancer. CRISP3 is expressed at low levels in normal human prostate and strongly upregulated in a fraction of prostate cancers. ${ }^{3-6}$ The clinical relevance of CRISP3 overexpression is controversially discussed. Some studies found CRISP3 overexpression to be associated with increased risk of tumor recurrence, ${ }^{7}$ unfavorable tumor phenotype, ${ }^{8,9}$ castration-resistant prostate cancer, and metastasis, ${ }^{9,10}$ whereas others could not find any clinically relevant associations. ${ }^{11,12}$

We and others recently identified a tight link of CRISP3 expression to positive $E R G$ fusion status in RNA expression screening studies. ${ }^{8,13}$ Associations of possible prognostic biomarkers with molecularly distinct tumor subtypes raise the question, whether their prognostic relevance may be limited to certain tumor subgroups. Such phenomenons could also explain the varying outcome of earlier studies involving relatively small patient sets.

To evaluate the potential clinical utility of CRISP3 measurement in prostate cancer we took advantage of a prostate cancer tissue microarray containing samples of $>10000$ patients with clinical follow-up information and an attached molecular database. Our data indicate a strong predilection of high-level CRISP3 expression in PTEN-deleted ERG fusionpositive prostate cancers. Accordingly, high CRISP3 expression is significantly linked to clinical outcome and tumor aggressiveness in prostate cancer.

\section{Materials and methods}

\section{Patients}

A set of tissue microarrays was made from 11152 prostatectomy specimens from patients undergoing surgery between 1992 and 2011 at the Department of Urology, and the Martini Clinics at the University Medical Center Hamburg-Eppendorf according to our institutional standard. ${ }^{14,15}$ The tissue microarray manufacturing process was described earlier in detail. ${ }^{16}$ In short, one $0.6 \mathrm{~mm}$ core was taken from a representative tissue block from each patient. The tissues were distributed among 24 tissue microarray blocks, each containing 144-522 cores. Clinical follow-up data were available for 9695 of 11152 arrayed tumors. Median follow-up was 36.8 months ranging from 1 to 228 months. PSA values were measured following surgery and recurrence was defined as a postoperative PSA of $0.2 \mathrm{ng} / \mathrm{ml}$ and increasing at first of appearance. The detailed composition of the tissue microarray and the
Table 1 Composition of the prognosis tissue microarray containing 11152 prostate cancer specimens

\begin{tabular}{|c|c|c|}
\hline & \multicolumn{2}{|c|}{ No. of patients } \\
\hline & $\begin{array}{l}\text { Study cohort on } \\
\text { tissue microarray }(\mathrm{n}=11152)\end{array}$ & $\begin{array}{c}\text { Biochemical } \\
\text { relapse among } \\
\text { categories }(\mathrm{n}=1824)\end{array}$ \\
\hline \multicolumn{3}{|c|}{ Follow-up (mo) } \\
\hline Mean & 53.4 & - \\
\hline Median & 36.8 & - \\
\hline \multicolumn{3}{|l|}{ Age (years) } \\
\hline$<50$ & 318 & 49 \\
\hline $50-60$ & 2768 & 460 \\
\hline $60-70$ & 6548 & 1081 \\
\hline$>70$ & 1439 & 232 \\
\hline \multicolumn{3}{|c|}{ Pretreatment PSA (ng/ml) } \\
\hline$<4$ & 1407 & 142 \\
\hline $4-10$ & 6735 & 827 \\
\hline $10-20$ & 2159 & 521 \\
\hline$>20$ & 720 & 309 \\
\hline \multicolumn{3}{|c|}{ pT category (AJCC 2002) } \\
\hline pT2 & 7370 & 570 \\
\hline рT3a & 2409 & 587 \\
\hline pT3b & 1262 & 618 \\
\hline $\mathrm{pT} 4$ & 63 & 49 \\
\hline \multicolumn{3}{|c|}{ Gleason grade } \\
\hline$\leq 3+3$ & 2859 & 193 \\
\hline $3+4$ & 1565 & 573 \\
\hline $4+3$ & 6183 & 849 \\
\hline$\geq 4+4$ & 482 & 208 \\
\hline \multicolumn{3}{|c|}{$p N$ category } \\
\hline pNo & 6117 & 1126 \\
\hline $\mathrm{pN}+$ & 561 & 291 \\
\hline \multicolumn{3}{|c|}{ Surgical margin } \\
\hline Negative & 8984 & 1146 \\
\hline Positive & 1970 & 642 \\
\hline
\end{tabular}

Abbreviation: AJCC, American Joint Committee on Cancer. NOTE: Numbers do not always add up to 11152 in the different categories because of cases with missing data.

attached histopathological and clinical data are outlined in Table 1. Presence or absence of cancer tissue was validated by immunohistochemical AMACR and 34BE12 analysis. ${ }^{17}$ The molecular database attached to this tissue microarray contained results on ERG expression in 8538, ERG break apart fluorescence in situ hybridization analysis in 1440 (extended from ${ }^{18}$ ), 5q21 deletions (CHD1) in 3023 (Burkhardt L, unpublished data), $6 q 15$ deletions (MAP3K7) in 2458 (Kluth et al; in press), ${ }^{19}$ PTEN deletions in $4088,{ }^{20}$ and $3 p 13$ deletions (FOXP1) in 1828 tumors (Krohn A, unpublished data).

\section{Immunohistochemistry}

Freshly cut tissue microarray sections were analyzed in 1 day and in one experiment. A polyclonal 
CRISP3 antibody (rabbit, Abcam; at 1/450 dilution; cat\#ab105951, Cambridge, UK) was used for detection of CRISP3 expression. Slides were deparaffinized and exposed to heat-induced antigen retrieval for $5 \mathrm{~min}$ in an autoclave at $121^{\circ} \mathrm{C}$ in $\mathrm{pH} 7.8$ buffer. Bound primary antibody was visualized using the DAKO EnVision Kit (Dako, Glostrup, Denmark). CRISP3 staining was evaluated according to the following scoring system as previously described: ${ }^{21-23}$ The staining intensity $(0,1+, 2+$, and $3+$ ) and the fraction of positive tumor cells were recorded for each tissue spot. A final score was built from these two parameters as follows: Negative: absence of CRISP3 staining; weak: intensity of $1+$ in $\leq 70 \%$ of tumor cells or staining intensity of $2+$ in $\leq 30 \%$ of tumor cells; moderate: intensity of $1+$ in $>70 \%$ of tumor cells, or staining intensity of $2+$ in $>30 \%$ but $\leq 70 \%$ of tumor cells or staining intensity of $3+\leq 30 \%$ of tumor cells; strong: intensity of $2+$ in $>70 \%$ of tumor cells or staining intensity of $3+$ in $>30 \%$ of tumor cells.

\section{Statistics}

For statistical analysis, the JMP 9.0 software (SAS Institute, NC, USA) was used. Contingency tables were calculated to study the association between CRISP3 expression and clinicopathological variables, and the $\chi^{2}$ - (Likelihood) test was used to find significant relationships. Kaplan-Meier curves were generated for PSA recurrence-free survival. The logRank test was applied to test the significance of differences between stratified survival functions. COX proportional hazards regression analysis was performed to test the statistical independence and significance between pathological, molecular, and clinical variables.

\section{Results}

\section{Technical Aspects}

CRISP3 analysis was successful in 9240/11152 arrayed cancers (82.9\%). Immunohistochemistry was non-informative in 1912 (17.1\%) tumors because lack of unequivocal tumor cells in the tissue spots or missing tissue spots on the tissue microarray section.

\section{CRISP3 Expression in Prostate Cancers}

CRISP3 protein was virtually not detectable in the epithelium of normal prostatic glands and stromal cells by immunohistochemistry. CRISP3 immunohistochemistry in 9240 informative tumors (82.9\%) revealed $1387(15 \%)$ tumors with weak, 790 $(8.5 \%)$ with moderate, and $662(7.2 \%)$ with strong staining, whereas 6401 (69.3\%) tumors did not show any staining (CRISP3 negative). Representative images of CRISP3 immunohistochemistry are shown in Figure 1. CRISP3 overexpression was significantly linked to advanced tumor stage, high Gleason grade, and positive surgical margins $(P<0.0001$; each) (Table 2).

\section{TMPRSS2-ERG Fusion Status and ERG Protein Expression}

The relationship of CRISP3 expression and 'fusiontype' prostate cancer was analyzed by two independent methods. There were subsets of 1216 cancers with available $E R G$ rearrangement data obtained by fluorescence in situ hybridization and 8032 cancers with ERG immunohistochemistry data for which CRISP3 data were also available. Strong CRISP3 staining was more frequent in ERG immunohistochemical positive $(461 / 3547 ; 13 \%)$ as compared with ERG immunohistochemical negative cancers (114/4485; 2.5\%, $P<0.0001$ ) (Figure 2a). Utilizing genomic ERG rearrangement data obtained by using an $E R G$ break apart probe yielded similar results. ${ }^{18}$ Strong CRISP3 staining was markedly more frequent in tumors with ERG rearrangement (67) $593 ; 11.3 \%)$ as compared with tumors without ERG rearrangement $(11 / 623 ; 1.7 \%) \quad(P<0.0001$; Figure 2b). A subset analysis in 3547 ERG fusionpositive cancers (Table 3) and 4485 ERG fusionnegative (Table 4) revealed that all associations of CRISP3 overexpression with unfavorable tumor phenotype held true for both ERG-negative and positive cancers. The associations of CRISP3 in ERG fusion-positive and -negative subgroups are summarized in Tables 3 and 4 .

\section{Associations of CRISP3 with Key Genomic Deletions in ERG Fusion-Positive and -Negative Prostate Cancers}

As several genomic deletions define distinct subgroups of ERG-negative and ERG-positive cancers, we next evaluated whether CRISP3 expression might be linked to one of these genomic aberrations. Data for these deletions were available to us from previous studies (PTEN, ${ }^{20}$ Kluth et $a l^{19}$; Burkhardt L, unpublished data; Krohn A, unpublished data), applying fluorescence in situ hybridization to parallel sections from our TMA. Deletion calling was at a threshold of $50 \%$ deleted tumor cells per spot in all these studies (PTEN, ${ }^{20}$ Kluth et al; ${ }^{19}$ Burkhardt L, unpublished data; Krohn A, unpublished data). The overall deletion frequency was $20.2 \%$ for PTEN, $16.5 \%$ for $3 p 13,18.5 \%$ for $6 q 15$, and $8.7 \%$ for $5 q 21$. The relationship of CRISP3 expression with these key deletions is shown in Figures 3-5 for all tumors and the subsets ERG fusion-negative and -positive prostate cancers.

As all these deletions are known to be strongly linked to $E R G$ fusion status, the overall association found for all these deletions with CRISP3 was expected. The subset analysis of ERG-positive 
a

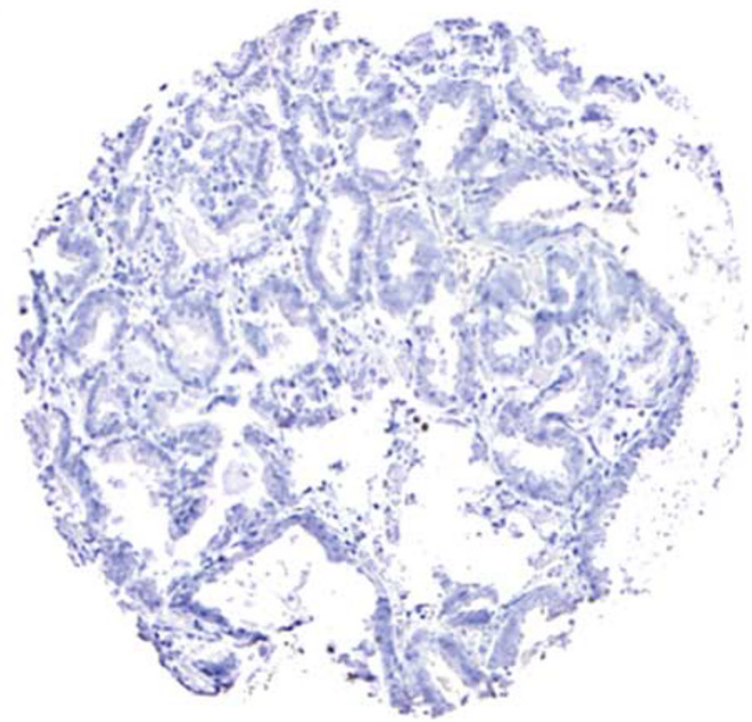

C

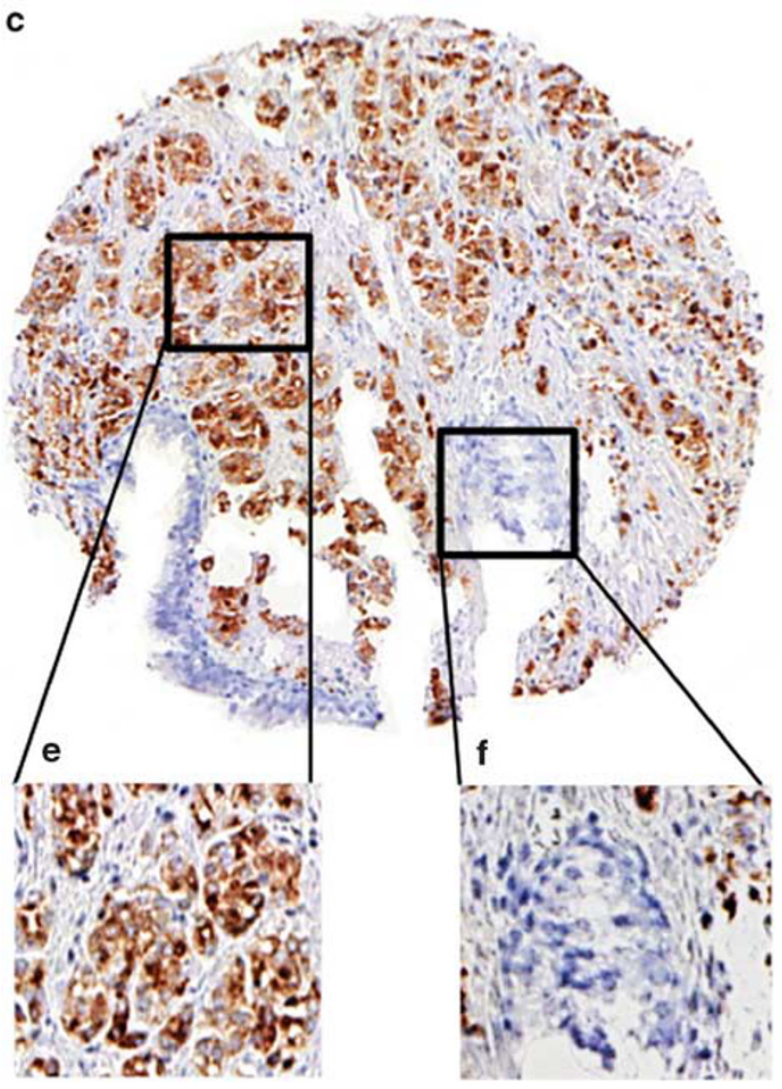

b

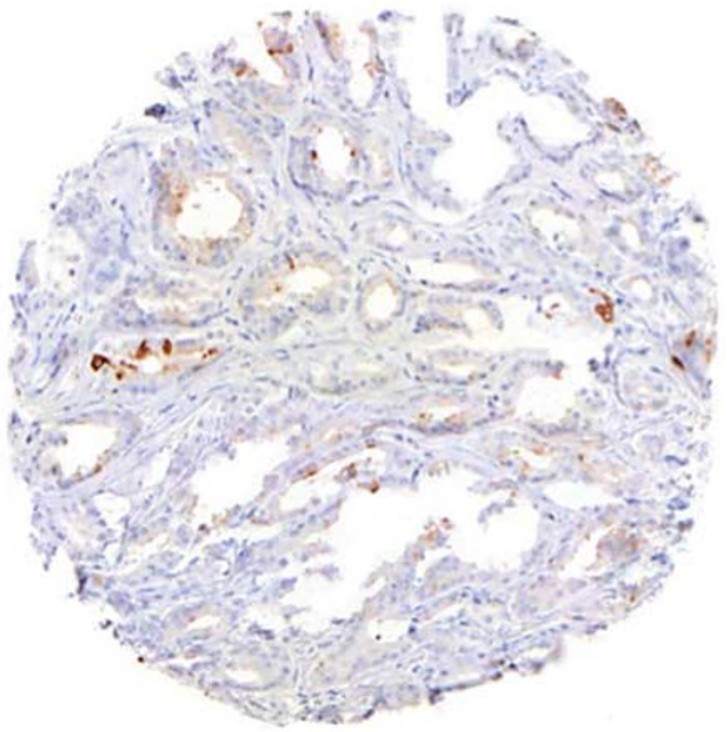

d

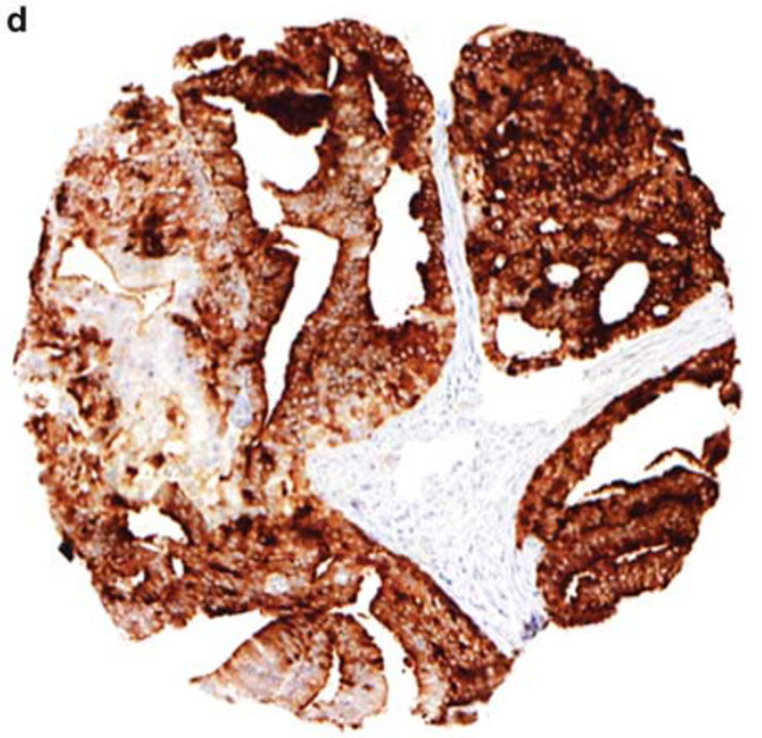

Figure 1 Representative pictures of CRISP3 immunostaining in prostate cancer. (a) Negative, (b) weak, (c) moderate, (d) strong staining, (e) magnification of moderate staining in cancer cells, and (f) magnification of negative staining of stromal tissue.

and ERG-negative cancers revealed, however, that only PTEN deletions were tightly linked to CRISP3 expression within these subgroups. Within ERG-positive cancers, strong CRISP3 immunostaining was found in 119 of 549 (21.7\%) PTEN deleted as compared with 119 of 1149
(10.4\%) cancers without PTEN deletion (Figure 4a). Within ERG-negative cancers, strong CRISP3 immunostaining was found in 9 of 246 $(3.7 \%)$ PTEN-deleted cancers as compared with 33 of $1481(2.2 \%)$ cancers without PTEN deletion $(P=0.0019$; Figure 5a). 
Table 2 Associations between CRISP3 immunostaining results and prostate cancer phenotype in all cancers

\begin{tabular}{|c|c|c|c|c|c|c|}
\hline \multirow{2}{*}{ Parameter } & \multirow{2}{*}{ n Evaluable } & \multicolumn{4}{|c|}{ CRISP3 immunohistochemistry result } & \multirow{2}{*}{$\mathrm{P}$ value } \\
\hline & & Negative (\%) & Weak $(\%)$ & Moderate (\%) & Strong (\%) & \\
\hline All cancers & 9240 & 69.3 & 15.0 & 8.5 & 7.2 & \\
\hline \multicolumn{7}{|l|}{ Tumor stage } \\
\hline pT2 & 5972 & 72.61 & 14.38 & 7.38 & 5.63 & \multirow[t]{4}{*}{$<0.0001$} \\
\hline рT3a & 2082 & 62.73 & 16.28 & 11.14 & 9.85 & \\
\hline pT3b & 1091 & 63.52 & 16.04 & 10.17 & 10.27 & \\
\hline pT4 & 54 & 59.26 & 18.52 & 9.26 & 12.96 & \\
\hline \multicolumn{7}{|c|}{ Gleason grade } \\
\hline$\leq 3+3$ & 2195 & 73.58 & 14.81 & 7.02 & 4.6 & \multirow[t]{4}{*}{$<0.0001$} \\
\hline $3+4$ & 5222 & 67.81 & 15.55 & 9.42 & 7.22 & \\
\hline $4+3$ & 1358 & 66.72 & 14.21 & 8.54 & 10.53 & \\
\hline$\geq 4+4$ & 414 & 72.22 & 12.08 & 6.52 & 9.18 & \\
\hline \multicolumn{7}{|c|}{ Lymph node metastasis } \\
\hline No & 5159 & 68.75 & 14.81 & 8.96 & 7.48 & \multirow[t]{2}{*}{0.016} \\
\hline $\mathrm{N}+$ & 485 & 62.68 & 17.32 & 9.07 & 10.93 & \\
\hline \multicolumn{7}{|c|}{ Surgical margin } \\
\hline Negative & 7362 & 70.13 & 15.12 & 8.22 & 6.53 & \multirow[t]{2}{*}{$<0.0001$} \\
\hline Positive & 1712 & 64.89 & 14.84 & 10.22 & 10.05 & \\
\hline
\end{tabular}
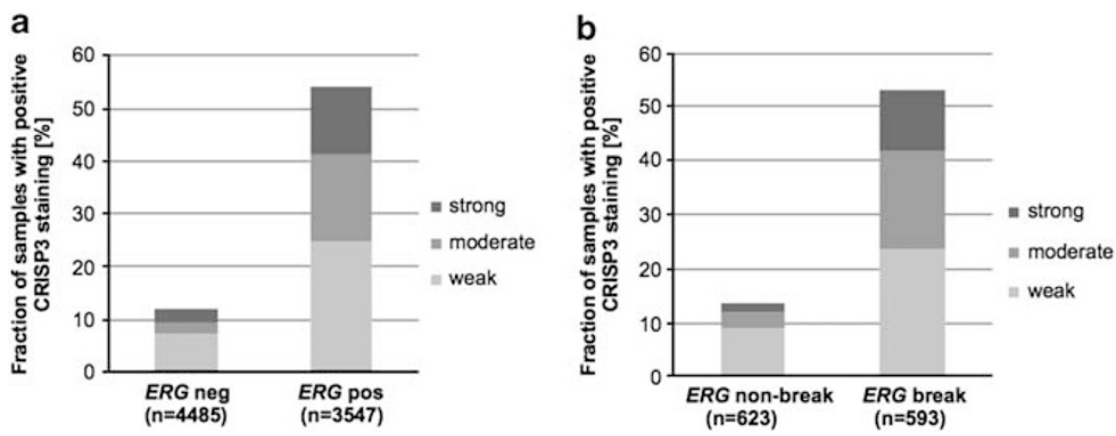

Figure 2 Association between positive CRISP3 immunostaining and $E R G$ fusion. ERG fusion was probed by both (a) immunohitochemistry and (b) fluorescence in situ hybridization analysis $(P<0.0001$; each).

\section{Prognostic Relevance of CRISP3 Expression}

Follow-up data were available from 8019 cancers. Strong CRISP3 staining was significantly linked to early biochemical recurrence in all cancers $(P=0.0013$, Figure 6a). This association also hold true in $3835 E R G$ fusion-negative $(P=0.004$, Figure 6b) and in $3080 E R G$ fusion-positive cancers $(P=0.0318$, Figure 6c). A combined analysis of PTEN deletion and CRISP3 expression status revealed that the prognostic impact of CRISP3 expression was mainly driven by its association with PTEN deletion. CRISP3 expression lost its prognostic relevance in subsets of 2428 PTEN-normal $(P=0.1796$, Figure $6 \mathrm{~d})$ and $751 \quad P T E N$-deleted cancers $(P=0.5291$, Figure 6e). Multivariate analysis including only PTEN and CRISP3 status revealed a strong prognostic impact of PTEN deletions on outcome $(P<0.0001)$ whereas CRISP3 expression provided no additional prognostic impact
$(P=0.6014)$. Multivariate analysis including also pT stage, Gleason grade, and preoperative PSA values revealed independent prognostic relevance for tumor stage $(P<0.0001)$, Gleason grade $(P<0.0001)$, PSA $(P<0.0001)$, and $P T E N$-deletion $(P=0.001)$, but not for CRISP3 expression $(P=0.618)$.

\section{Discussion}

The results of our study indicate that strong CRISP3 expression is highly linked to ERG fusion-type prostate cancer and-within this subgroup-to presence of PTEN inactivation. Accordingly, high CRISP3 expression is also tied to adverse tumor phenotype and early PSA recurrence.

Earlier studies using microarrays had found massive CRISP3 overexpression in a subset of prostate cancers. Ernst et $a{ }^{3}{ }^{3}$ described CRISP3 to be overexpressed $>20$-fold in microdissected 
Table 3 Associations between CRISP3 immunostaining results and ERG-positive prostate cancer phenotype

\begin{tabular}{|c|c|c|c|c|c|c|}
\hline \multirow{2}{*}{ Parameter } & \multirow{2}{*}{ n Evaluable } & \multicolumn{4}{|c|}{ CRISP3 immunohistochemistry result } & \multirow{2}{*}{$\mathrm{P}$ value } \\
\hline & & Negative (\%) & Weak $(\%)$ & Moderate (\%) & Strong $(\%)$ & \\
\hline All cancers & 3547 & 45.9 & 24.8 & 16.7 & 13.0 & \\
\hline \multicolumn{7}{|l|}{ Tumor stage } \\
\hline pT2 & 2120 & 48.96 & 24.76 & 15.52 & 10.75 & \multirow[t]{4}{*}{$<0.0001$} \\
\hline pT3a & 933 & 40.94 & 24.76 & 18.54 & 15.76 & \\
\hline pT3b & 452 & 41.59 & 24.12 & 16.59 & 17.7 & \\
\hline pT4 & 23 & 26.09 & 43.48 & 13.04 & 17.39 & \\
\hline \multicolumn{7}{|c|}{ Gleason grade } \\
\hline$\leq 3+3$ & 776 & 52.19 & 24.87 & 14.18 & 8.76 & \multirow[t]{4}{*}{$<0.0001$} \\
\hline $3+4$ & 2112 & 43.8 & 25.14 & 18.04 & 13.02 & \\
\hline $4+3$ & 512 & 43.36 & 24.41 & 14.06 & 18.16 & \\
\hline$\geq 4+4$ & 124 & 48.39 & 19.35 & 13.71 & 18.55 & \\
\hline \multicolumn{7}{|c|}{ Lymph node metastasis } \\
\hline No & 2047 & 44.11 & 24.72 & 17.49 & 13.68 & \multirow[t]{2}{*}{0.1631} \\
\hline $\mathrm{N}+$ & 205 & 43.9 & 25.37 & 12.68 & 18.05 & \\
\hline \multicolumn{7}{|c|}{ Surgical margin } \\
\hline Negative & 2776 & 47.12 & 24.71 & 16.07 & 12.1 & \multirow[t]{2}{*}{0.001} \\
\hline Positive & 703 & 40.11 & 25.18 & 17.92 & 16.79 & \\
\hline
\end{tabular}

Table 4 Associations between CRISP3 immunostaining results and ERG-negative prostate cancer phenotype

\begin{tabular}{|c|c|c|c|c|c|c|}
\hline \multirow{2}{*}{ Parameter } & \multirow{2}{*}{ n Evaluable } & \multicolumn{4}{|c|}{ CRISP3 immunohistochemistry result } & \multirow{2}{*}{$\mathrm{P}$ value } \\
\hline & & Negative (\%) & Weak $(\%)$ & Moderate (\%) & Strong (\%) & \\
\hline All cancers & 4485 & 87.9 & 7.1 & 2.4 & 2.5 & \\
\hline \multicolumn{7}{|l|}{ Tumor stage } \\
\hline pT2 & 2999 & 89.63 & 6.97 & 1.67 & 1.73 & $<0.0001$ \\
\hline pT3а & 916 & 85.26 & 6.99 & 3.93 & 3.82 & \\
\hline pT3b & 523 & 82.6 & 8.41 & 4.4 & 4.59 & \\
\hline pT4 & 28 & 85.71 & 0 & 3.57 & 10.71 & \\
\hline \multicolumn{7}{|c|}{ Gleason grade } \\
\hline$\leq 3+3$ & 962 & 90.64 & 6.96 & 1.56 & 0.83 & $<0.0001$ \\
\hline $3+4$ & 2539 & 88.54 & 7.17 & 2.13 & 2.17 & \\
\hline $4+3$ & 707 & 83.45 & 6.51 & 4.67 & 5.37 & \\
\hline$\geq 4+4$ & 254 & 83.07 & 8.66 & 3.15 & 5.12 & \\
\hline \multicolumn{7}{|c|}{ Lymph node metastasis } \\
\hline No & 2646 & 88.21 & 6.73 & 2.49 & 2.57 & 0.0017 \\
\hline $\mathrm{N}+$ & 227 & 79.3 & 9.69 & 4.85 & 6.17 & \\
\hline \multicolumn{7}{|c|}{ Surgical margin } \\
\hline Negative & 3572 & 88.3 & 7.42 & 2.21 & 2.07 & $<0.0001$ \\
\hline Positive & 821 & 85.75 & 5.97 & 3.65 & 4.63 & \\
\hline
\end{tabular}

prostate cancer as compared with adjacent normal prostate epithelium. Others found a 20-2000-fold upregulation of CRISP3 mRNA in cancerous prostate. $^{5,6}$ As low-level CRISP3 expression does also occur in normal prostate epithelium, ${ }^{4}$ our immunohistochemistry protocol was designed to identify high CRISP3 expressers and not to detect tissues containing any level of CRISP3 protein. Utilizing such an experimental setup, we identified $16 \%$ of prostate cancers with moderate-to-strong CRISP3 expression, whereas the remaining $84 \%$ of tumors had weak or absent CRISP3 immunostaining. This fraction of cancers exhibiting high-level CRISP3 expression fits well with data by Hoogland et al describing $13 \%$ of their prostate cancers showing a striking CRISP3 positivity at low magnification in their tissue microarray study. ${ }^{11}$ Previous studies have reported higher rates of CRISP3 overexpression ranging between 19 and $96 \%$ of prostate cancers. ${ }^{3,7-9,12}$ We assume that these authors partly 

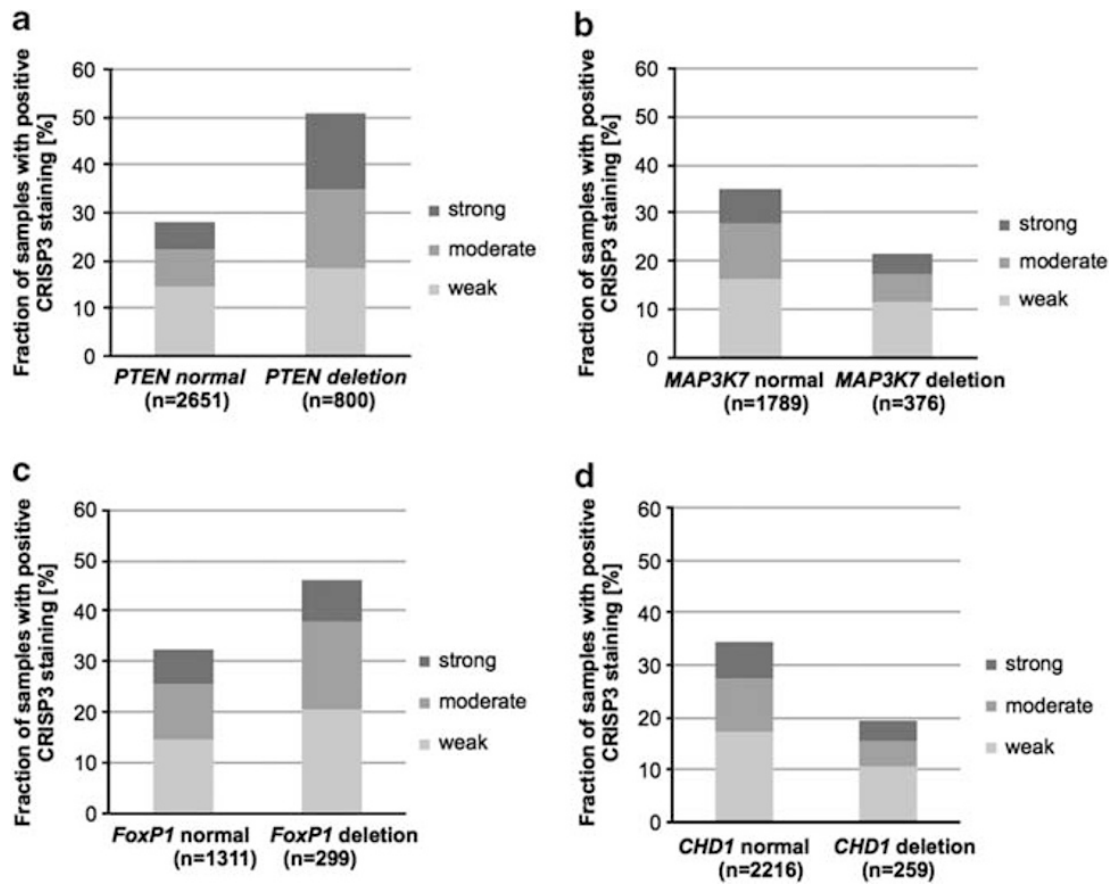

Figure 3 Association between positive CRISP3 immunostaining and (a) PTEN, (b) MAP3K7, (c) FOXP1, and (d) CHD1 deletion probed by fluorescence in situ hybridization analysis in all cancers $(P<0.0001$; each).
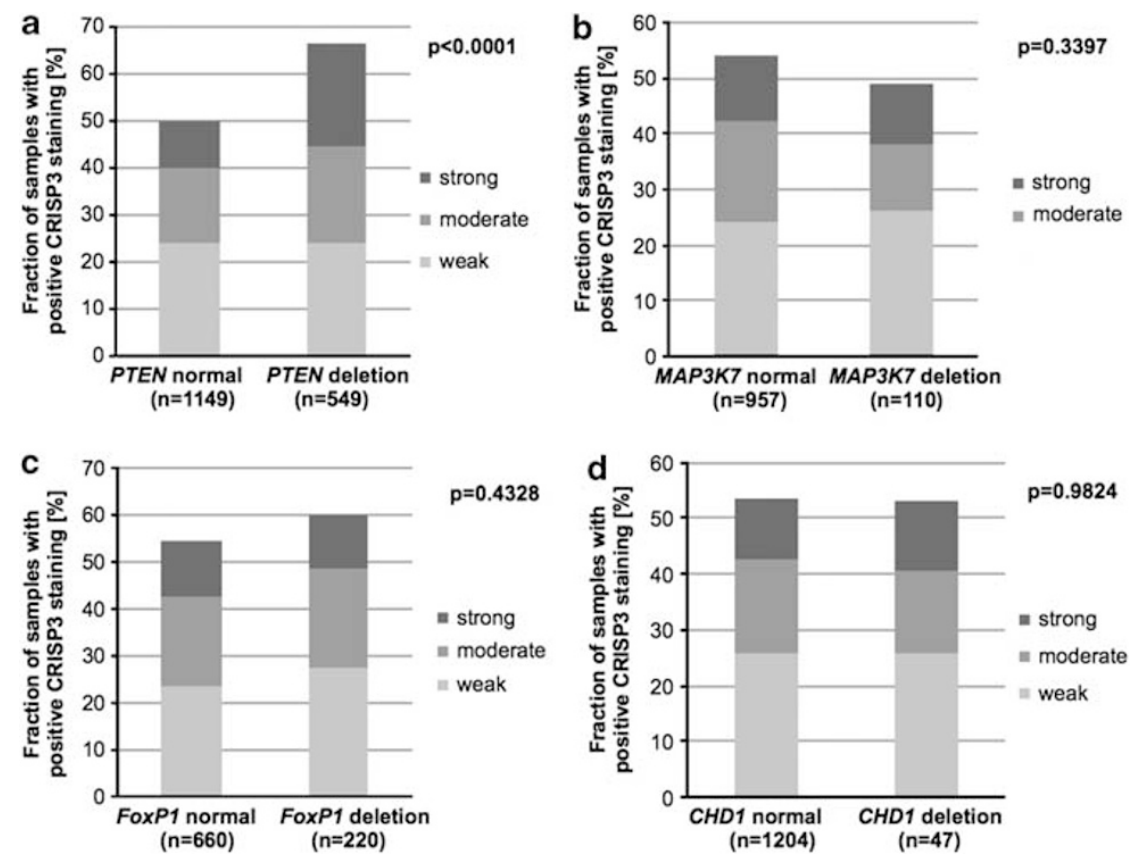

Figure 4 Association between positive CRISP3 immunostaining and (a) PTEN, (b) MAP3K7, (c) FOXP1, and (d) CHD1 deletion probed by fluorescence in situ hybridization analysis in ERG fusion-positive cancers.

used markedly more sensitive immunohistochemistry approaches than selected for our project. The relatively low rate of CRISP3 positivity seen in our study cannot be caused by representative issues of our tissue microarray approach. Earlier immunohistochemical studies using tissue microarrays reported $74-98 \%$ CRISP3 positivity, ${ }^{7,9,12}$ including one study analyzing a subset of the tissue microarrays employed in this project. $^{12}$ It is possible, that automated TMA reading with unusually sensitive thresholds has contributed to the high expression rate. ${ }^{12}$

High CRISP3 expression was strongly linked to $E R G$ fusion-positive cancers. Finding this association 

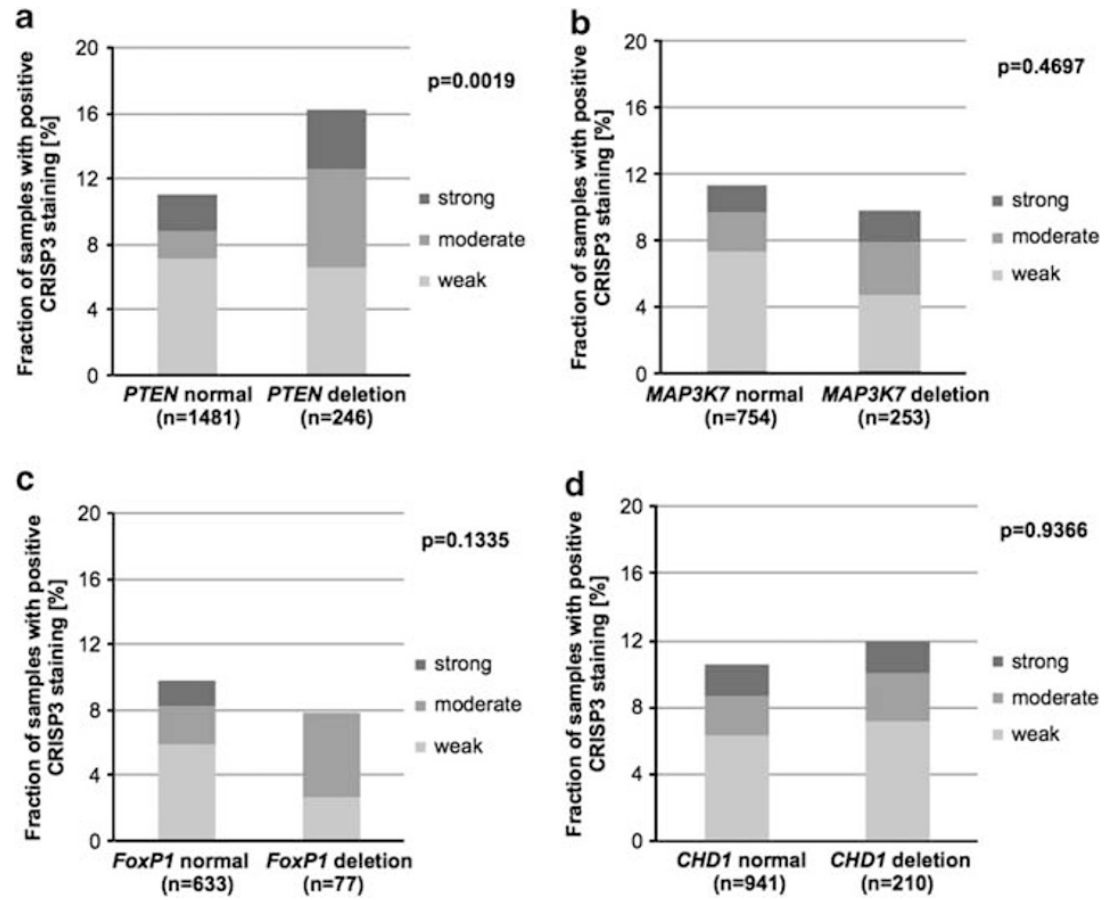

Figure 5 Association between positive CRISP3 immunostaining and (a) PTEN, (b) MAP3K7, (c) FOXP1, and (d) CHD1 deletion probed by fluorescence in situ hybridization analysis in ERG fusion-negative cancers.
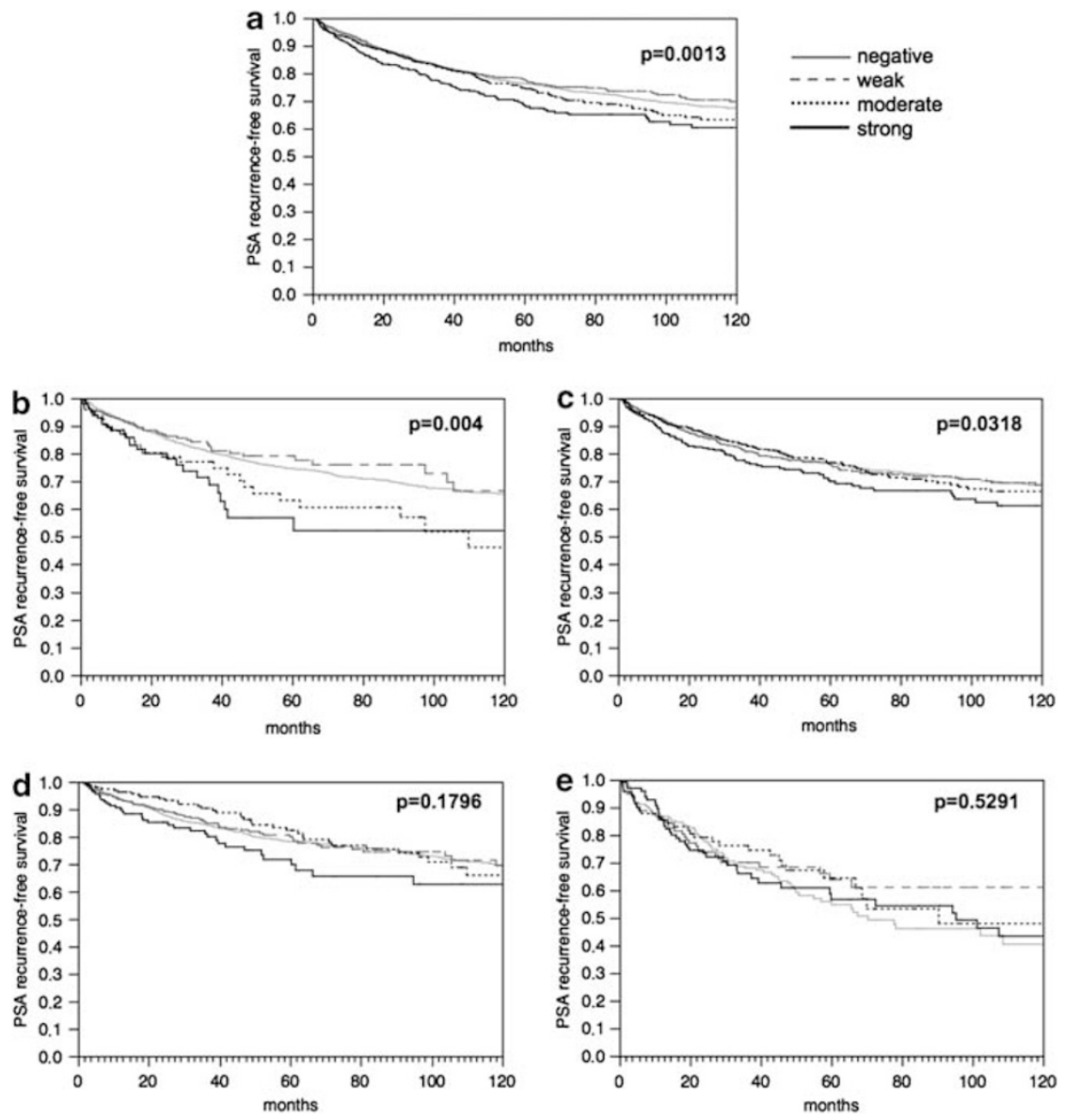

Figure 6 Association between CRISP3 immunostaining intensity and biochemical recurrence in (a) all cancers ( $n=8019$ ), (b) $E R G$ fusion-negative cancers $(n=3835)$, (c) $E R G$ fusion-positive cancers $(n=3080)$, (d) cancers without PTEN deletion $(n=2428)$, and (e) cancers with PTEN deletion $(n=751)$. 
by two independent approaches for $E R G$ fusion detection (immunohistochemistry/fluorescence in situ hybridization) largely excludes a false-positive association due to inefficient immunostaining in a subset of damaged non-reactive tissues. Earlier data derived from mRNA expression screening studies by Brase et $a l^{13}$ and Ribeiro et $a l^{8}$ had already indicated an association between ERG fusion and CRISP3 expression in prostate cancer. Activation of ERG expression, mostly caused by TMPRSS2:ERG gene fusion, results in aberrant activation of different signaling cascades including upregulation of several metabolic enzymes, as well as extracellular/ transmembrane proteins involved in cell adhesion, matrix remodeling, and signal transduction. ${ }^{13,24-26}$ Our study identifies CRISP3 as another extracellular protein, which is strongly upregulated in $E R G$ fusionpositive cancers. The functional role of CRISP3 in prostate cancer development and/or progression is unclear. It may be speculated, that CRISP3 exerts a role in cancer through beta-microseminoprotein (MSMB), a protein with proapoptotic activity and tumor inhibitory effects in prostate cancer cell lines. ${ }^{27-29}$ CRISP3 forms complexes with MSMB. The MSMB is strongly downregulated in prostate cancer and thus prostate cancer progression may be critically affected by the amount of unbound CRISP3. ${ }^{30}$

Earlier genomic studies had identified chromosomal deletions that were tightly linked to either $E R G$ positive or $E R G$-negative prostate cancer. In particular, deletions at $3 p 13$ and of PTEN were found to be associated with ERG-positive and $5 q 21$ and $6 q 15$ to $E R G$-negative cancers. ${ }^{31-33}$ Our comparison of CRISP3 expression with these genomic deletions revealed, that high-level CRISP3 expression is particularly linked to ERG-positive/PTEN-deleted prostate cancer. These data may indicate, that either activation of a pathway that also induces CRISP3 overexpression may facilitate PTEN inactivation or else, that PTEN inactivation-at least in ERG positive cancers-may facilitate development of certain molecular features eventually leading to CRISP3 overexpression.

As $22 \%$ of all strong CRISP3 expressing ERGpositive prostate cancers had PTEN inactivation-a major negative prognosticator in prostate cancer-in our study, it was not surprising, that high-level CRISP3 expression was strongly associated with unfavorable tumor phenotype and early PSA recurrence in this study. Earlier studies had also suggested a negative prognostic role of high CRISP3 levels in serum and prostate cancer tissue. ${ }^{7,9}$ Using immunohistochemistry on biopsies Bjartell et al ${ }^{9}$ described CRISP3 as overexpressed in high-grade (Gleason scores 4/5) prostate cancer. It was also found in one study, that patients with CRISP3 overexpression had a slightly higher risk of recurrence after radical prostatectomy. ${ }^{7}$ Using a consecutive series of 200 prostatectomy samples, Ribeiro et $a l^{8}$ found upregulation of CRISP3 mRNA to be associated with stage pT3. The clinical utility of CRISP3 expression analysis remains questionable, as our multivariable analysis including also stage, grade, preoperative PSA values, and PTEN deletions did not suggest an independent prognostic role of CRISP3 overexpression. Other authors also failed to find an independent prognostic role of CRISP3 expression in prostate cancer. ${ }^{8,10-12}$

In summary, high CRISP3 expression identifies a small subset of prostate cancer harboring $E R G$ fusion and PTEN deletion, which is clinically characterized by unfavorable tumor phenotype and early PSA recurrence.

\section{Acknowledgements}

We thank Christina Koop, Julia Schumann, Sünje Seekamp, and Inge Brandt for excellent technical assistance.

\section{Disclosure/conflict of interest}

The authors declare no conflict of interest.

\section{References}

1 Jemal A, Siegel R, Xu J, et al. Cancer statistics, 2010. CA Cancer J Clin 2010;60:277-300.

2 Gibbs GM, Roelants K, O’Bryan MK. The CAP superfamily: cysteine-rich secretory proteins, antigen 5 , and pathogenesis-related 1 proteins-roles in reproduction, cancer, and immune defense. Endocr Rev 2008;29: 865-897.

3 Ernst T, Hergenhahn M, Kenzelmann M, et al. Decrease and gain of gene expression are equally discriminatory markers for prostate carcinoma: a gene expression analysis on total and microdissected prostate tissue. Am J Pathol 2002;160:2169-2180.

4 Kratzschmar J, Haendler B, Eberspaecher U, et al. The human cysteine-rich secretory protein (CRISP) family. Primary structure and tissue distribution of CRISP-1, CRISP-2 and CRISP-3. Eur J Biochem 1996;236: 827-836.

5 Asmann YW, Kosari F, Wang K, et al. Identification of differentially expressed genes in normal and malignant prostate by electronic profiling of expressed sequence tags. Cancer Res 2002;62:3308-3314.

6 Kosari F, Asmann YW, Cheville JC, et al. Cysteine-rich secretory protein-3: a potential biomarker for prostate cancer. Cancer Epidemiol Biomarkers Prev 2002;11: 1419-1426.

7 Bjartell AS, Al-Ahmadie H, Serio AM, et al. Association of cysteine-rich secretory protein 3 and betamicroseminoprotein with outcome after radical prostatectomy. Clin Cancer Res 2007;13:4130-4138.

8 Ribeiro FR, Paulo P, Costa VL, et al. Cysteine-rich secretory protein-3 (CRISP3) is strongly up-regulated in prostate carcinomas with the TMPRSS2-ERG fusion gene. PLoS One 2011;6:e22317.

9 Bjartell A, Johansson R, Bjork T, et al. Immunohistochemical detection of cysteine-rich secretory protein 3 in tissue and in serum from men with cancer or benign 
enlargement of the prostate gland. Prostate 2006;66: 591-603.

10 Dahlman A, Edsjo A, Hallden C, et al. Effect of androgen deprivation therapy on the expression of prostate cancer biomarkers MSMB and MSMB-binding protein CRISP3. Prostate Cancer Prostatic Dis 2010;13:369-375.

11 Hoogland AM, Dahlman A, Vissers KJ, et al. Cysteinerich secretory protein 3 and beta-microseminoprotein on prostate cancer needle biopsies do not have predictive value for subsequent prostatectomy outcome. BJU Int 2011;108:1356-1362.

12 Dahlman A, Rexhepaj E, Brennan DJ, et al. Evaluation of the prognostic significance of MSMB and CRISP3 in prostate cancer using automated image analysis Mod Pathol 2011;24:708-719.

13 Brase JC, Johannes M, Mannsperger $\mathrm{H}$, et al. TMPRSS2-ERG -specific transcriptional modulation is associated with prostate cancer biomarkers and TGF-beta signaling. BMC Cancer 2011;11:507.

14 Schlomm T, Heinzer $\mathrm{H}$, Steuber $\mathrm{T}$, et al. Full functional-length urethral sphincter preservation during radical prostatectomy. Eur Urol 2011;60:320-329.

15 Schlomm T, Tennstedt P, Huxhold C, et al. Neurovascular structure-adjacent frozen-section examination (NeuroSAFE) increases nerve-sparing frequency and reduces positive surgical margins in open and robotassisted laparoscopic radical prostatectomy: experience after 11069 consecutive patients. Eur Urol 2012; 62:333-340.

16 Kononen J, Bubendorf L, Kallioniemi A, et al. Tissue microarrays for high-throughput molecular profiling of tumor specimens. Nat Med 1998;4:844-847.

17 Minner S, Jessen B, Stiedenroth L, et al. Low level HER2 overexpression is associated with rapid tumor cell proliferation and poor prognosis in prostate cancer. Clin Cancer Res 2010;16:1553-1560.

18 Minner S, Enodien M, Sirma H, et al. ERG status is unrelated to PSA recurrence in radically operated prostate cancer in the absence of antihormonal therapy. Clin Cancer Res 2011;17:5878-5888.

19 Kluth M, Hesse J, Heinl A, et al. Genomic deletion of MAP3K7 at 6q12-22 is associated with early PSA recurrence in prostate cancer and absence of TMPRSS2-ERG fusions. Mod Pathol 2012 (in press).

20 Krohn A, Diedler T, Burkhardt L, et al. Genomic deletion of pten is associated with tumor progression and early PSA recurrence in ERG fusion-positive and fusion-negative prostate cancer. Am J Pathol 2012; 181:401-412.
21 Clauditz TS, Gontarewicz A, Lebok P, et al. Epidermal growth factor receptor (EGFR) in salivary gland carcinomas: potentials as therapeutic target. Oral Oncol 2012;48:991-996.

22 Muller J, Ehlers A, Burkhardt L, et al. Loss of $\mathrm{p}$ (Ser2448) -mTOR expression is linked to adverse prognosis and tumor progression in ERG-fusion-positive cancers. Int J Cancer 2012; doi:10.1002/ijc.27768.

23 Minner S, Wittmer C, Graefen M, et al. High level PSMA expression is associated with early PSA recurrence in surgically treated prostate cancer. Prostate 2011;71:281-288.

24 Tomlins SA, Laxman B, Varambally S, et al. Role of the TMPRSS2-ERG gene fusion in prostate cancer. Neoplasia 2008;10:177-188.

25 Gupta S, Iljin K, Sara H, et al. FZD4 as a mediator of ERG oncogene-induced WNT signaling and epithelialto-mesenchymal transition in human prostate cancer cells. Cancer Res 2010;70:6735-6745.

26 Hermans KG, Boormans JL, Gasi D, et al. Overexpression of prostate-specific TMPRSS2(exon 0)-ERG fusion transcripts corresponds with favorable prognosis of prostate cancer. Clin Cancer Res 2009;15:6398-6403.

27 Garde SV, Basrur VS, Li L, et al. Prostate secretory protein (PSP94) suppresses the growth of androgenindependent prostate cancer cell line (PC3) and xenografts by inducing apoptosis. Prostate 1999;38: 118-125.

28 Shukeir N, Arakelian A, Kadhim S, et al. Prostate secretory protein PSP-94 decreases tumor growth and hypercalcemia of malignancy in a syngenic in vivo model of prostate cancer. Cancer Res 2003;63: 2072-2078.

29 Pathak BR, Breed AA, Nakhawa VH, et al. Growth inhibition mediated by PSP94 or CRISP-3 is prostate cancer cell line specific. Asian J Androl 2010;12: 677-689.

30 Chan PS, Chan LW, Xuan JW, et al. In situ hybridization study of PSP94 (prostatic secretory protein of 94 amino acids) expression in human prostates. Prostate 1999;41:99-109.

31 Taylor BS, Schultz N, Hieronymus H, et al. Integrative genomic profiling of human prostate cancer. Cancer Cell 2010;18:11-22.

32 Berger MF, Lawrence MS, Demichelis F, et al. The genomic complexity of primary human prostate cancer. Nature 2010;470:214-220.

33 Lapointe J, Li C, Giacomini CP, et al. Genomic profiling reveals alternative genetic pathways of prostate tumorigenesis. Cancer Res 2007;67:8504-8510. 